\title{
DIAGONAL MULTILINEAR OPERATORS ON KÖTHE SEQUENCE SPACES
}

\author{
VERÓNICA DIMANT AND ROMÁN VILLAFAÑE
}

\begin{abstract}
We analyze the interplay between maximal/minimal/adjoint ideals of multilinear operators (between sequence spaces) and their associated Köthe sequence spaces. We establish relationships with spaces of multipliers and apply these results to describe diagonal multilinear operators from Lorentz sequence spaces. We also define and study some properties of the ideal of $(E, p)$-summing multilinear mappings, a natural extension of the linear ideal of absolutely $(E, p)$-summing operators.
\end{abstract}

\section{INTRODUCTION}

Trying to describe the connections between different ideals of linear operators (and the internal structure of them), it began, in the 70's, the study of diagonal linear operators on $\ell_{p}$ spaces with the work of Carl [10], König [27] and Pietsch [35]. By means of limit orders they have compared different ideals of linear operators and described their diagonal elements. Next, this research continued in the context of Köthe sequence spaces, leading to the so called multipliers. This notion has its root in harmonic analysis where it has appeared within the study of Fourier series and Fourier transformation. Later, it has also been employed in many other contexts as, closer to our framework, Banach function spaces and Banach sequence spaces [14, 5, 2, 24, 25, 26].

The concept of ideal of multilinear operators was also introduced by Pietsch in [36] and it has been developed by several authors since then. Even if the multilinear theory has its roots in the linear one, it had its own development that led to different situations involving new interesting techniques. Some usual linear ideals (absolutely $p$-summing operators, for instance) have many natural diverse extensions to the multilinear setting which enrich the theory by showing interesting situations that do not appear in the linear context (see, for instance [34, 6, 33]). We refer to [19, 20] for general results about ideals of multilinear mappings. For a presentation of the multilinear theory focused in the interplay with polynomials and holomorphic mappings the books [16] and [32] are the classical references.

2010 Mathematics Subject Classification. 46A45, 47L22,47H60.

Key words and phrases. Multilinear ideals, Köthe sequence spaces, Diagonal multilinear operators.

Both authors were partially supported by CONICET PIP 2014-0483 and ANPCyT PICT 2015-2299. The second author was also partially supported by UBACyT 20020130100474 BA. 
The introduction of limit orders for studying ideals of multilinear forms appeared in [7] and similar methods were used for general sequence spaces in [8]. There, it was defined the Köthe sequence space associated to an ideal of multilinear forms acting on Köthe sequence spaces. Later, in [9], this kind of study reached vector valued multilinear ideals between $\ell_{p}$-spaces.

Here, we propose a more general approach of the relationship between ideals of multilinear operators (acting on Köthe sequence spaces) and their respective associated sequence spaces. In particular, we analyze if for a maximal (minimal) ideal its associated sequence space is also maximal (minimal). In addition, we relate the sequence spaces associated to an ideal and its adjoint.

The spaces of multipliers appear to give us new descriptions of our sequence spaces associated to multilinear ideals. As an application, we can characterize diagonal multilinear operators from Lorentz sequence spaces.

In the final section, we define the ideal of $(E, p)$-summing multilinear mappings, as a natural extension of the linear ideal of absolutely $(E, p)$-summing operators. We obtain some properties of this multilinear ideal by means of our previous results on associated sequence spaces.

\section{Preliminaries}

Throughout the paper we will use standard notation of the Banach space theory. We will consider complex Banach spaces $E, F, \ldots$ and their duals will be denoted by $E^{\prime}, F^{\prime}, \ldots$ We will write $E=F$ if they are topologically isomorphic and $E \stackrel{1}{=} F$ if they are isometrically isomorphic. The symbol $\stackrel{1}{\hookrightarrow}$ means an isometric injection and the symbol $\stackrel{\leq 1}{\hookrightarrow}$ means a norm one inclusion (not necessarily isometric).

Sequences of complex numbers will be denoted by $x=(x(k))_{k=1}^{\infty}$, where each $x(k) \in \mathbb{C}$. By a Köthe sequence space (also known as Banach sequence space) we mean a Banach space $E \subseteq \mathbb{C}^{\mathbb{N}}$ such that $\ell_{1} \stackrel{\leq 1}{\longrightarrow} E \stackrel{\leq 1}{\hookrightarrow} \ell_{\infty}$ and with the normal property: if $x \in \mathbb{C}^{\mathbb{N}}$ and $y \in E$ satisfy $|x(k)| \leq|y(k)|$ for all $k \in \mathbb{N}$ then $x \in E$ and $\|x\| \leq\|y\|$. Note that in a Köthe sequence space $E$, given $x \in E$ and a sequence of complex numbers $s$ with $|s(k)|=1$ for all $k \in \mathbb{N}$, we should have $s \cdot x \in E$ and $\|s \cdot x\|=\|x\|$ (where the product is coordinatewise). For each $N \in \mathbb{N}$, we consider the $N$-dimensional truncation $E_{N}:=\operatorname{span}\left\{e_{1}, \ldots, e_{N}\right\}$ (where $e_{n}$ denotes the $n$-th canonical unit vector: $e_{n}(k)=\delta_{n, k}$ for all $k$ ). The canonical inclusion and projection will be denoted by $i_{N}: E_{N} \stackrel{1}{\hookrightarrow} E$ and $\pi_{N}: E \rightarrow E_{N}$.

The Köthe dual of a Köthe sequence space $E$, defined as

$$
E^{\times}:=\left\{z \in \mathbb{C}^{\mathbb{N}}: \sum_{j \in \mathbb{N}}|z(j) \cdot x(j)|<\infty \text { for all } x \in E\right\},
$$


is a Köthe sequence space with the norm

$$
\|z\|_{E^{\times}}:=\sup _{\|x\|_{E} \leq 1} \sum_{j \in \mathbb{N}}|z(j) \cdot x(j)|=\sup _{\|x\|_{E} \leq 1}\|z \cdot x\|_{\ell_{1}} .
$$

It is well known (see, for example, [3, Lemma 2.8]) that $z \in E^{\times}$if and only if the series $\sum_{j \in \mathbb{N}} z(j) \cdot x(j)$ converges for all $x \in E$. Also, $\|z\|_{E^{\times}}=\sup _{\|x\|_{E} \leq 1}\left|\sum_{j \in \mathbb{N}} z(j) \cdot x(j)\right|$. Note that $\left(E_{N}\right)^{\prime} \stackrel{1}{=}\left(E^{\times}\right)_{N}$ holds for every $N$. In the same way that we define the Köthe dual, we can considerate $\left(E^{\times}\right)^{\times}=E^{\times \times}$and we say that $E$ is Köthe reflexive if $E^{\times \times} \stackrel{1}{=} E$.

Following [29, 1.d], a Köthe sequence space $E$ is said to be $r$-convex (with $1 \leq r<\infty$ ) if there exists a constant $\kappa>0$ such that for any choice $x_{1}, \ldots, x_{m} \in E$ we have

$$
\left\|\left(\left(\sum_{j=1}^{m}\left|x_{j}(k)\right|^{r}\right)^{1 / r}\right)_{k=1}^{\infty}\right\|_{E} \leq \kappa\left(\sum_{j=1}^{m}\left\|x_{j}\right\|_{E}^{r}\right)^{1 / r} .
$$

We denote by $\mathbf{M}^{(r)}(E)$ the smallest constant which satisfies the inequality.

The minimal kernel of a Köthe sequence space $E$ is defined as the set

$$
E^{\text {min }}:=\left\{x \in \ell_{\infty}: x=y \cdot z \text { with } y \in E \text { and } z \in c_{0}\right\}
$$

which is also a Köthe sequence space if we endow it with the norm

$$
\|x\|_{E^{\text {min }}}=\inf \left\{\|y\|_{E} \cdot\|z\|_{\ell_{\infty}}: x=y \cdot z \quad \text { with } y \in E \text { and } z \in c_{0}\right\} .
$$

The maximal hull of a Köthe sequence space $E$ is defined as the set

$$
E^{\max }:=\left\{x \in \ell_{\infty}: x \cdot z \in E \text { for all } z \in c_{0}\right\},
$$

which results a Köthe sequence space if the norm is given by

$$
\|x\|_{E^{\max }}=\sup _{z \in B_{c_{0}}}\|x \cdot z\|_{E} .
$$

A Köthe sequence space $E$ is said to be maximal if $E \stackrel{1}{=} E^{\text {max }}$ and minimal if $E \stackrel{1}{=} E^{m i n}$. For example, a Köthe dual $E^{\times}$is always maximal.

For a detailed study and general facts about Köthe sequence spaces, see [28, 29].

The space of continuous linear operators between two Banach spaces $E$ and $F$ will be denoted by $\mathcal{L}(E ; F)$ and the space of continuous $n$-linear mappings from $E_{1} \times \cdots \times E_{n}$ to $F$ by $\mathcal{L}\left(E_{1}, \ldots, E_{n} ; F\right)$. This is a Banach space with the usual sup norm, given by $\|T\|:=\sup \left\{\left\|T\left(x_{1}, \ldots, x_{n}\right)\right\|_{F}:\left\|x_{i}\right\|_{E_{i}} \leq 1, i=1, \ldots n\right\}$. If $E_{1}=\cdots=E_{n}=E$ we will write $\mathcal{L}\left({ }^{n} E ; F\right)$ and whenever $F=\mathbb{C}$ we will simply write $\mathcal{L}\left(E_{1}, \ldots, E_{n}\right)$ or $\mathcal{L}\left({ }^{n} E\right)$.

Ideals of multilinear forms and multilinear operators were introduced by Pietsch in [36]. Let us recall the definition. An ideal of multilinear operators $\mathfrak{A}$ is a subclass of $\mathcal{L}$, the class continuous multilinear operators, such that, for any Banach spaces $E_{1}, \ldots, E_{n}$ and $F$ the set

$$
\mathfrak{A}\left(E_{1}, \ldots, E_{n} ; F\right)=\mathfrak{A} \cap \mathcal{L}\left(E_{1}, \ldots, E_{n} ; F\right)
$$


satisfies

(1) If $S, T \in \mathfrak{A}\left(E_{1}, \ldots, E_{n} ; F\right)$, then $S+T \in \mathfrak{A}\left(E_{1}, \ldots, E_{n} ; F\right)$.

(2) If $T \in \mathfrak{A}\left(E_{1}, \ldots, E_{n} ; F\right)$ and $B_{i} \in \mathcal{L}\left(G_{i}, E_{i}\right)$ for $i=1, \ldots, n$ and $A \in \mathcal{L}(F ; H)$, then $A \circ T \circ\left(B_{1}, \ldots B_{n}\right) \in \mathfrak{A}\left(G_{1}, \ldots, G_{n} ; H\right)$.

(3) The mapping $\left(x_{1}, \ldots, x_{n}\right) \mapsto \gamma_{1}\left(x_{1}\right) \cdots \gamma_{n}\left(x_{n}\right) \cdot f$ belongs to $\mathfrak{A}\left(E_{1}, \ldots, E_{n} ; F\right)$ for any $\gamma_{1} \in E_{1}^{\prime}, \ldots, \gamma_{n} \in E_{n}^{\prime}$ and $f \in F$.

An ideal of multilinear operators is called normed if for each $E_{1}, \ldots, E_{n}$ and $F$ there is a norm $\|\cdot\|_{\mathfrak{A}\left(E_{1}, \ldots, E_{n} ; F\right)}$ in $\mathfrak{A}\left(E_{1}, \ldots, E_{n} ; F\right)$ such that

(1) $\left\|\left(x_{1}, \ldots, x_{n}\right) \mapsto \gamma_{1}\left(x_{1}\right) \cdots \gamma_{n}\left(x_{n}\right) \cdot f\right\|_{\mathfrak{A}\left(E_{1}, \ldots, E_{n} ; F\right)}=\left\|\gamma_{1}\right\| \cdots\left\|\gamma_{n}\right\| \cdot\|f\|$.

(2) $\left\|A \circ T \circ\left(B_{1}, \ldots, B_{n}\right)\right\|_{\mathfrak{A}\left(G_{1}, \ldots, G_{n} ; H\right)} \leq\|A\| \cdot\|T\|_{\mathfrak{A}\left(E_{1}, \ldots, E_{n} ; F\right)} \cdot\left\|B_{1}\right\| \cdots\left\|B_{n}\right\|$.

If $\mathfrak{A}\left(E_{1}, \ldots, E_{n} ; F\right)$ is complete for every Banach spaces $E_{1}, \ldots, E_{n}, F$ we say that $\mathfrak{A}$ is a Banach ideal of multilinear operators (or just, a Banach multilinear ideal).

The minimal kernel of $\mathfrak{A}$ is defined as the composition ideal $\mathfrak{A}^{\text {min }}:=\overline{\mathfrak{F}} \circ \mathfrak{A} \circ(\overline{\mathfrak{F}}, \ldots, \overline{\mathfrak{F}})$, where $\overline{\mathfrak{F}}$ stands for the ideal of approximable operators (i. e. the closure of the ideal of finite rank linear operators). In other words, a multilinear operator $T$ belongs to $\mathfrak{A}^{\text {min }}\left(E_{1}, \ldots, E_{n} ; F\right)$ if it admits a factorization

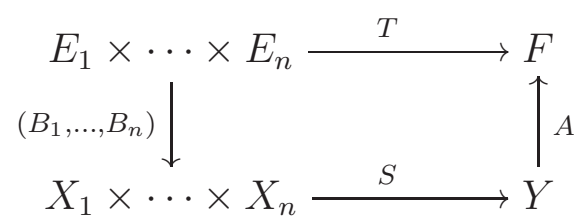

where $A, B_{1}, \ldots, B_{n} \in \overline{\mathfrak{F}}$ and $S \in \mathfrak{A}\left(X_{1}, \ldots, X_{n} ; Y\right)$. The $\mathfrak{A}^{\text {min }}$-norm of $T$ is given by $\|T\|_{\mathfrak{A} \text { min }}:=\inf \left\{\|A\| \cdot\|S\|_{\mathfrak{A}} \cdot\left\|B_{1}\right\| \cdots\left\|B_{n}\right\|\right\}$, where the infimum runs over all possible factorizations as in (11). $\mathfrak{A}^{\text {min }}$ is the smallest Banach multilinear ideal whose norm coincides with $\|\cdot\|_{\mathfrak{A}}$ over finite dimensional spaces. This and other properties of $\mathfrak{A}^{\text {min }}$ can be found in [17. An ideal of multilinear operators is said to be minimal if $\left(\mathfrak{A},\|\cdot\|_{\mathfrak{A}}\right) \stackrel{1}{=}\left(\mathfrak{A}^{\text {min }},\|\cdot\|_{\mathfrak{A}^{\text {min }}}\right)$.

If $\mathfrak{A}$ is a normed ideal of $n$-linear operators, the maximal hull $\mathfrak{A}^{\text {max }}$ of $\mathfrak{A}$ is defined as the class of all $n$-linear operators $T$ such that

$$
\|T\|_{\mathfrak{A}^{\max }}:=\sup \left\{\left\|Q_{L}^{Y} \circ T \circ\left(I_{M_{1}}^{X_{1}}, \ldots, I_{M_{n}}^{X_{n}}\right)\right\|_{\mathfrak{A}}: M_{i} \in F I N\left(X_{i}\right), L \in \operatorname{COFIN}(Y)\right\}
$$

is finite, where $I_{M}^{X}: M \rightarrow X$ is the inclusion from $M$ into $X, Q_{L}^{Y}: Y \rightarrow Y / L$ is the projection of $Y$ over $Y / L$ and $F I N(X)(C O F I N(X))$ represents the class of subspaces of $X$ of finite dimension (codimension). $\mathfrak{A}^{\max }$ is always complete and it is the largest ideal whose norm coincides with $\|\cdot\|_{\mathfrak{A}}$ over finite dimensional spaces. A normed ideal $\mathfrak{A}$ is called maximal if $\left(\mathfrak{A},\|\cdot\|_{\mathfrak{A}}\right) \stackrel{1}{=}\left(\mathfrak{A}^{\max },\|\cdot\|_{\mathfrak{A}^{\max }}\right)$.

If $\mathfrak{A}$ is an ideal of multilinear operators, its associated tensor norm is the unique finitely generated tensor norm $\alpha$, of order $n+1$, satisfying

$$
\mathfrak{A}\left(M_{1}, \ldots, M_{n} ; N\right) \stackrel{1}{=}\left(M_{1}^{\prime} \otimes \cdots \otimes M_{n}^{\prime} \otimes N ; \alpha\right)
$$


for every finite dimensional spaces $M_{1}, \ldots, M_{n}, N$. In that case we write $\mathfrak{A} \sim \alpha$. A detailed study of tensor norms and their relationship with linear/multilinear ideals can be found in [12, 17, 18, 19, 20]. Note that $\mathfrak{A}, \mathfrak{A}^{\max }$ and $\mathfrak{A}^{\text {min }}$ have the same associated tensor norm since they coincide isometrically on finite dimensional spaces.

Given a normed ideal $\mathfrak{A}$ associated to a finitely generated tensor norm $\alpha$, its adjoint ideal $\mathfrak{A}^{*}$ is defined by

$$
\mathfrak{A}^{*}\left(E_{1}, \ldots, E_{n} ; F\right):=\left(E_{1} \otimes \cdots \otimes E_{n} \otimes F^{\prime}, \alpha\right)^{\prime} \cap \mathcal{L}\left(E_{1}, \ldots, E_{n} ; F\right) .
$$

The adjoint ideal is called dual ideal in [17]. The tensor norm associated to $\mathfrak{A}^{*}$ is denoted by $\alpha^{\prime}$. It is well known, by the representation theorem for maximal ideals [20, Section 4.5], that $\mathfrak{A}^{*}$ is always maximal and $\mathfrak{A}^{* *} \stackrel{1}{=} \mathfrak{A}^{\text {max }}$.

Recall that a multilinear operator $T \in \mathcal{L}\left(E_{1}, \ldots, E_{n} ; F\right)$ is (Grothendieck) integral if there exists a regular $F^{\prime \prime}$-valued Borel measure $\mu$, of bounded variation on $\left(B_{E_{1}^{\prime}} \times \cdots \times\right.$ $\left.B_{E_{n}^{\prime}}, w^{*}\right)$ such that

$$
T\left(x_{1}, \ldots, x_{n}\right)=\int_{B_{E_{1}^{\prime}} \times \cdots \times B_{E_{n}^{\prime}}}\left(x_{1}^{\prime}\left(x_{1}\right)\right) \cdots\left(x_{n}^{\prime}\left(x_{n}\right)\right) d \mu\left(x_{1}^{\prime}, \ldots, x_{n}^{\prime}\right)
$$

for every $x_{k} \in E_{k}$. The space of Grothendieck integral $n$-linear operators is denoted by $\mathcal{I}\left(E_{1}, \ldots, E_{n} ; F\right)$ and the integral norm of a multilinear operator $T$ is defined as $\inf \{\|\mu\|\}$, where the infimum runs over all the measures $\mu$ representing $T$. This ideal is maximal and its adjoint is the ideal $\mathcal{L}$ of all continuous multilinear mappings.

\section{Interplay BetWeEn AN IDEAL AND its ASSOCIATED SEQUENCE SPACE}

For Köthe sequence spaces $E$ and $F$, an $n$-linear operator $T \in \mathcal{L}\left({ }^{n} E ; F\right)$ is said to be diagonal if there exists a bounded sequence $\alpha=(\alpha(k))_{k} \in \mathbb{C}^{\mathbb{N}}$ such that for all $x_{1}, \ldots, x_{n} \in E$ we can write

$$
T\left(x_{1}, \ldots, x_{n}\right)=\alpha \cdot x_{1} \cdots x_{n}=\sum_{k \in \mathbb{N}} \alpha(k) \cdot x_{1}(k) \cdots x_{n}(k) \cdot e_{k} .
$$

In this case, we say that $T$ is the diagonal multilinear operator associated with $\alpha$ and we denote it $T_{\alpha}$. Given $\mathfrak{A}$, an ideal of multilinear operators, we define the sequence space associated with $\mathfrak{A}$ as

$$
\ell_{\boldsymbol{n}}(\mathfrak{A} ; E, F):=\left\{\alpha \in \ell_{\infty}: T_{\alpha} \in \mathfrak{A}\left({ }^{n} E ; F\right)\right\} .
$$

This is a Köthe sequence space endowed with the norm $\|\alpha\|_{\ell_{n}(\mathfrak{A} ; E, F)}=\left\|T_{\alpha}\right\|_{\mathfrak{A}\left({ }^{n} E ; F\right)}$. When $F=\mathbb{C}$ we simply write $\boldsymbol{\ell}_{\boldsymbol{n}}(\mathfrak{A} ; E)$.

The following finite-dimensional identifications are easy to check. They will enable us to prove a duality result next.

$$
\begin{gathered}
\boldsymbol{\ell}_{n}(\mathfrak{A} ; E, F)_{N} \stackrel{1}{=} \boldsymbol{\ell}_{\boldsymbol{n}}\left(\mathfrak{A} ; E_{N}, F_{N}\right) \stackrel{1}{=} \boldsymbol{\ell}_{\boldsymbol{n}}\left(\mathfrak{A} ; E_{N}^{\times \times}, F_{N}^{\times \times}\right) \stackrel{1}{=} \boldsymbol{\ell}_{\boldsymbol{n}}\left(\mathfrak{A} ; E^{\times \times}, F^{\times \times}\right)_{N} . \\
\boldsymbol{\ell}_{\boldsymbol{n}}\left(\mathfrak{A} ; E_{N}, F_{N}\right) \stackrel{1}{=} \boldsymbol{\ell}_{\boldsymbol{n}}\left(\mathfrak{A}^{\max } ; E_{N}, F_{N}\right) \stackrel{1}{=} \boldsymbol{\ell}_{\boldsymbol{n}}\left(\mathfrak{A}^{m i n} ; E_{N}, F_{N}\right) .
\end{gathered}
$$


Our aim is to analyze first the relationship between minimal or maximal ideals with their respective associated sequence spaces and later the interplay between the sequence space associated with an ideal and its adjoint.

In [8, Proposition 5.5 and 5.6] it is proved that if $\mathfrak{A}$ is a maximal ideal of multilinear forms (scalar valued multilinear operators), then $\boldsymbol{\ell}_{\boldsymbol{n}}(\mathfrak{A} ; E) \stackrel{1}{=} \boldsymbol{\ell}_{\boldsymbol{n}}\left(\mathfrak{A} ; E^{\times \times}\right)$and $\boldsymbol{\ell}_{\boldsymbol{n}}(\mathfrak{A} ; E) \stackrel{1}{=}$ $\boldsymbol{\ell}_{\boldsymbol{n}}\left(\mathfrak{A}^{*} ; E^{\times}\right)^{\times}$. In both cases the key of the proofs is the use of [8, Lemma 5.4], which is a version of the Density Lemma [12, 13.4] for diagonal multilinear forms. So, we begin by proving a new version of this Lemma in our vector-valued context and then we can establish some similar results to those given above.

Lemma 2.1. Let $\mathfrak{A}$ be a maximal ideal of n-linear operators and let $E$ and $F$ be Köthe sequence spaces. For a sequence $\alpha$, suppose that there exists a constant $C>0$ such that the projection $\pi_{N}(\alpha)$ satisfies $\left\|\pi_{N}(\alpha)\right\|_{\ell_{\boldsymbol{n}}\left(\mathfrak{A} ; E_{N}, F\right)} \leq C$ for all $N \in \mathbb{N}$. Then, $\alpha \in \boldsymbol{\ell}_{\boldsymbol{n}}\left(\mathfrak{A} ; E, F^{\times \times}\right)$ and $\|\alpha\|_{\ell_{n}\left(\mathfrak{a} ; E, F^{\times \times}\right)} \leq C$.

In other words, if $T_{\pi_{N}(\alpha)} \in \mathfrak{A}\left({ }^{n} E_{N} ; F\right)$ has $\mathfrak{A}$-norm less than or equal to $C$ for all $N \in \mathbb{N}$ then $T_{\alpha} \in \mathfrak{A}\left({ }^{n} E ; F^{\times \times}\right)$with $\mathfrak{A}$-norm less than or equal to $C$.

Proof. Since $\mathfrak{A}$ is maximal, by [20, Theorem 4.5] there exists a finitely generated tensor norm $\nu$ of order $n+1$ such that $\mathfrak{A}\left({ }^{n} E ; F^{\prime \prime}\right) \stackrel{1}{=}\left(\otimes^{n} E \otimes F^{\prime} ; \nu\right)^{\prime}$. Then, the ball $B_{\mathfrak{A}\left({ }^{n} E ; F^{\prime \prime}\right)}$ is weak-star compact. Thus, the set $\left(J_{F} \circ T_{\pi_{N}(\alpha)} \circ\left(\pi_{N}, \ldots, \pi_{N}\right)\right)_{N}$, which is contained in the ball $C \cdot B_{\mathfrak{A}\left({ }^{(n} E ; F^{\prime \prime}\right)}$, has a weak-star accumulation point $\Phi \in C \cdot B_{\mathfrak{A}\left({ }^{n} E ; F^{\prime \prime}\right)}$. This mapping should satisfy $\Phi\left(x_{1}, \ldots, x_{n}\right)\left(e_{k}^{\prime}\right)=\alpha(k) \cdot x_{1}(k) \cdots x_{n}(k)$, for all $x_{1}, \ldots, x_{n} \in E, e_{k}^{\prime} \in F^{\prime}$.

On the other hand, the canonical mapping $\xi: F^{\prime \prime} \rightarrow F^{\times \times}$is well defined and has norm less than or equal to 1 . Hence, $\xi \circ \Phi$ belongs to $C \cdot B_{\mathfrak{A}\left({ }^{n} E ; F^{\times \times}\right)}$and

$$
\xi \circ \Phi\left(x_{1}, \ldots, x_{n}\right)=\left(\Phi\left(x_{1}, \ldots, x_{n}\right)\left(e_{k}^{\prime}\right)\right)_{k}=\left(\alpha(k) \cdot x_{1}(k) \cdots x_{n}(k)\right)_{k} .
$$

This says that $\xi \circ \Phi$ coincides with the mapping $T_{\alpha}$. In consequence, $T_{\alpha} \in \mathfrak{A}\left({ }^{n} E ; F^{\times \times}\right)$ with $\left\|T_{\alpha}\right\|_{\mathfrak{A}\left({ }^{n} E, F^{\times \times}\right)} \leq C$.

In particular, if $F$ is maximal and $E_{0}=\operatorname{span}\left\{e_{j}: j \in \mathbb{N}\right\}$, given a diagonal multilinear operator $T_{\alpha}: E_{0} \times \cdots \times E_{0} \rightarrow F$ such that their truncated operators satisfy $\left\|T_{\pi_{N}(\alpha)}\right\|_{\mathfrak{A}\left({ }^{n} E_{N} ; F\right)} \leq C$ for all $N \in \mathbb{N}$, it follows that $T_{\alpha} \in \mathfrak{A}\left({ }^{n} E ; F\right)$ with $\left\|T_{\alpha}\right\|_{\mathfrak{A}\left({ }^{n} E ; F\right)} \leq C$.

In order to prove the following result, recall the well known characterization of the maximal hull of a sequence space: $x \in E^{\max }$ if and only if $\sup _{N \in \mathbb{N}}\left\|\pi_{N}(x)\right\|_{E}$ is finite, and the norm is given by this supremum. In other words, to ensure that a sequence space $E$ is maximal it is enough to show that if $\left\|\pi_{N}(x)\right\|_{E} \leq C$ for all $N \in \mathbb{N}$, then $x \in E$ with $\|x\|_{E} \leq C$.

Proposition 2.2. Let $\mathfrak{A}$ be an ideal of n-linear operators and let $E$ and $F$ be Köthe sequence spaces.

(i) If $\mathfrak{A}$ is maximal, then $\boldsymbol{\ell}_{\boldsymbol{n}}\left(\mathfrak{A} ; E, F^{\times \times}\right)$is a maximal Köthe sequence space and $\boldsymbol{\ell}_{\boldsymbol{n}}\left(\mathfrak{A} ; E, F^{\times \times}\right) \stackrel{1}{=} \boldsymbol{\ell}_{\boldsymbol{n}}\left(\mathfrak{A} ; E^{\times \times}, F^{\times \times}\right)$. 
(ii) $\boldsymbol{\ell}_{\boldsymbol{n}}(\mathfrak{A} ; E, F)^{\max } \stackrel{1}{=} \boldsymbol{\ell}_{\boldsymbol{n}}\left(\mathfrak{A}^{\max } ; E, F^{\times \times}\right)$.

Proof. (i) Suppose that $\left\|\pi_{N}(\alpha)\right\|_{\ell_{n}\left(\mathfrak{A} ; E, F^{\times \times}\right)} \leq C$ for all $N \in \mathbb{N}$. By identity (2), we have that $\left\|\pi_{N}(\alpha)\right\|_{\ell_{n}\left(\mathfrak{A} ; E_{N}, F^{\times \times}\right)}=\left\|\pi_{N}(\alpha)\right\|_{\ell_{n}\left(\mathfrak{A} ; E, F^{\times x}\right)} \leq C$. Then, by Lemma 2.1, $\alpha \in$ $\boldsymbol{\ell}_{\boldsymbol{n}}\left(\mathfrak{A} ; E, F^{\times \times}\right)$with $\|\alpha\|_{\ell_{\boldsymbol{n}}\left(\mathfrak{A} ; E, F^{\times \times}\right)} \leq C$. So, $\boldsymbol{\ell}_{\boldsymbol{n}}\left(\mathfrak{A} ; E, F^{\times \times}\right)$is maximal and the same is true for $\boldsymbol{\ell}_{\boldsymbol{n}}\left(\mathfrak{A} ; E^{\times \times}, F^{\times \times}\right)$. Again, identity (2), assures, for each sequence $\alpha$, that $\left\|\pi_{N}(\alpha)\right\|_{\ell_{n}\left(\mathfrak{A} ; E, F^{\times \times}\right)}=\left\|\pi_{N}(\alpha)\right\|_{\ell_{n}\left(\mathfrak{A} ; E^{\times \times}, F^{\times \times}\right)}$, for all $N \in \mathbb{N}$. Therefore, $\boldsymbol{\ell}_{\boldsymbol{n}}\left(\mathfrak{A} ; E, F^{\times \times}\right) \stackrel{1}{=}$ $\boldsymbol{\ell}_{\boldsymbol{n}}\left(\mathfrak{A} ; E^{\times \times}, F^{\times \times}\right)$.

(ii) For a sequence $\alpha$, by identities (21) and (3),$\left\|\pi_{N}(\alpha)\right\|_{\ell_{n}(\mathfrak{A} ; E, F)}=\left\|\pi_{N}(\alpha)\right\|_{\ell_{n}\left(\mathfrak{A} \max ; E, F^{\times \times}\right)}$ and $\boldsymbol{\ell}_{\boldsymbol{n}}(\mathfrak{A} ; E, F)^{\text {max }} \stackrel{1}{=} \boldsymbol{\ell}_{\boldsymbol{n}}\left(\mathfrak{A}^{\max } ; E, F^{\times \times}\right)^{\max }$. Then, applying item (i) we have that $\boldsymbol{\ell}_{\boldsymbol{n}}\left(\mathfrak{A}^{\text {max }} ; E, F^{\times \times}\right)^{\text {max }} \stackrel{1}{=} \boldsymbol{\ell}_{\boldsymbol{n}}\left(\mathfrak{A}^{\text {max }} ; E, F^{\times \times}\right)$, which completes the proof.

Remark 2.3. By the previous proposition, if both the ideal $\mathfrak{A}$ and the sequence space $F$ are maximal, then the sequence space $\boldsymbol{\ell}_{\boldsymbol{n}}(\mathfrak{A} ; E, F)$ is maximal. Note that the condition over $F$ is necessary for $\boldsymbol{\ell}_{\boldsymbol{n}}(\mathfrak{A} ; E, F)$ to be maximal. Indeed, if $\mathfrak{A}=\mathcal{L}, E=\ell_{\infty}$ and $F=c_{0}$, it follows that $\boldsymbol{\ell}_{\boldsymbol{n}}\left(\mathcal{L} ; \ell_{\infty}, c_{0}\right)=c_{0}$, which obviously is not a maximal sequence space.

Now, we turn to look into the minimal hull. Recall that a sequence space $E$ is minimal if and only if for all $x \in E,\left\|\left(\pi_{N}^{E}-I_{E}\right)(x)\right\|_{E} \rightarrow 0$, or equivalently, $\pi_{N}^{E}$ tends to $I_{E}$ over compact sets.

Proposition 2.4. Let $\mathfrak{A}$ be an ideal of n-linear operators and let $E$ and $F$ be Köthe sequence spaces.

(i) If $\mathfrak{A}$ is a minimal ideal and $F$ is a minimal Köthe sequence space, then $\boldsymbol{\ell}_{\boldsymbol{n}}(\mathfrak{A} ; E, F)$ is a minimal sequence space.

(ii) $\boldsymbol{\ell}_{\boldsymbol{n}}(\mathfrak{A} ; E, F)^{\text {min }} \stackrel{1}{=} \boldsymbol{\ell}_{\boldsymbol{n}}\left(\mathfrak{A}^{\text {min }} ; E, F^{\text {min }}\right)$.

Proof. (i) Let $\alpha \in \boldsymbol{\ell}_{\boldsymbol{n}}(\mathfrak{A} ; E, F)$. Since $\mathfrak{A}$ is minimal, there exist approximable linear operators $A_{1}, \ldots, A_{n}, B$ and $S \in \mathfrak{A}$ such that $T_{\alpha}=B \circ S \circ\left(A_{1}, \ldots, A_{n}\right)$. Then,

$$
\left(\pi_{N}^{F}-I_{F}\right) \circ T_{\alpha}=\left(\pi_{N}^{F}-I_{F}\right) \circ B \circ S \circ\left(A_{1}, \ldots, A_{n}\right) \in \mathfrak{A}\left({ }^{n} E ; F\right)
$$

and $\left\|\left(\pi_{N}^{F}-I_{F}\right) \circ T_{\alpha}\right\|_{\mathfrak{A}\left({ }^{n} E ; F\right)} \leq\left\|\left(\pi_{N}^{F}-I_{F}\right) \circ B\right\| \cdot\|S\|_{\mathfrak{A}} \cdot\left\|A_{1}\right\| \cdots\left\|A_{n}\right\|$. Now, the mapping $B \in \overline{\mathfrak{F}}$, which has $F$ as its target space, is compact and $\pi_{N}^{F}$ tends to $I_{F}$ over compact sets (because $F$ is minimal), therefore $\left\|\left(\pi_{N}^{F}-I_{F}\right) \circ B\right\|$ tends to zero. In consequence, $\left\|\left(\pi_{N}^{F}-I_{F}\right) \circ T_{\alpha}\right\|_{\mathfrak{A}\left({ }^{n} E ; F\right)}$ tends to zero also and $\boldsymbol{\ell}_{\boldsymbol{n}}(\mathfrak{A} ; E, F)$ is minimal.

(ii) For $\alpha \in \boldsymbol{\ell}_{\boldsymbol{n}}(\mathfrak{A} ; E, F)^{\text {min }}$, the norm $\left\|\pi_{N}(\alpha)-\alpha\right\|_{\ell_{\boldsymbol{n}}(\mathfrak{A} ; E, F)}$ tends to zero. By the identities (2) and (3) we have $\left\|\pi_{N}(\alpha)\right\|_{\ell_{n}(\mathfrak{A} ; E, F)}=\left\|\pi_{N}(\alpha)\right\|_{\ell_{\boldsymbol{n}}\left(\mathfrak{A}_{\text {min }} ; E, F^{\text {min }}\right)}$. Then, $\left(\pi_{N}(\alpha)\right)_{N}$ is a Cauchy sequence in the Banach sequence space $\boldsymbol{\ell}_{\boldsymbol{n}}\left(\mathfrak{A}^{\text {min }} ; E, F^{\text {min }}\right)$ and hence it converges to a sequence in $\boldsymbol{\ell}_{\boldsymbol{n}}\left(\mathfrak{A}^{\text {min }} ; E, F^{\text {min }}\right)$ that coincides with $\alpha$ coordinate by coordinate. In 
other words, this says that $\alpha \in \boldsymbol{\ell}_{\boldsymbol{n}}\left(\mathfrak{A}^{\text {min }} ; E, F^{\text {min }}\right)$ and

$$
\begin{aligned}
\|\alpha\|_{\ell_{\boldsymbol{n}}\left(\mathfrak{A}^{\text {min }} ; E, F^{\text {min }}\right)} & \leq\left\|\pi_{N}(\alpha)-\alpha\right\|_{\ell_{\boldsymbol{n}}\left(\mathfrak{A}^{\text {min }} ; E, F^{m i n}\right)}+\left\|\pi_{N}(\alpha)\right\|_{\ell_{\boldsymbol{n}}\left(\mathfrak{A}^{\text {min }} ; E, F^{\text {min }}\right)} \\
& =\left\|\pi_{N}(\alpha)-\alpha\right\|_{\boldsymbol{\ell}_{\boldsymbol{n}}\left(\mathfrak{A}^{\text {min }} ; E, F^{m i n}\right)}+\left\|\pi_{N}(\alpha)\right\|_{\ell_{\boldsymbol{n}}(\mathfrak{A} ; E, F)} \rightarrow\|\alpha\|_{\boldsymbol{\ell}_{\boldsymbol{n}}(\mathfrak{A} ; E, F)^{\text {min }}} .
\end{aligned}
$$

The reverse inclusion holds by item (i).

Now, we analyze the relationship between the sequence space associated to an ideal with the associated to its adjoint. Note that when we have a finite dimensional space, its Köthe dual and its classical dual coincide. Moreover, if we call $\nu$ the tensor norm associated to the ideal $\mathfrak{A}$, we have,

$$
\mathfrak{A}^{*}\left({ }^{n} E_{N}^{\times} ; F_{N}^{\times}\right) \stackrel{1}{=}\left(\otimes^{n} E_{N}^{\times} \otimes F_{N}^{\times \times}, \nu\right)^{\prime} \stackrel{1}{=} \mathfrak{A}\left({ }^{n} E_{N}^{\times \times} ; F_{N}^{\times \times}\right)^{\prime} \stackrel{1}{=} \mathfrak{A}\left({ }^{n} E_{N} ; F_{N}\right)^{\prime} .
$$

The duality is given in the following way: if $T \in \mathfrak{A}\left({ }^{n} E_{N} ; F_{N}\right)$ and $S \in \mathfrak{A}^{*}\left({ }^{n} E_{N}^{\times} ; F_{N}^{\times}\right)$, we can represent them as $T=\sum_{i} x_{i, 1}^{\times} \otimes \cdots \otimes x_{i, n}^{\times} \otimes y_{i}$ and $S=\sum_{j} x_{j, 1} \otimes \cdots \otimes x_{j, n} \otimes y_{j}^{\times}$. Then,

$$
\langle S, T\rangle=\sum_{i, j} x_{i, 1}^{\times}\left(x_{j, 1}\right) \cdots x_{i, n}^{\times}\left(x_{j, n}\right) \cdot y_{j}^{\times}\left(y_{i}\right)
$$

It is plain that $|\langle S, T\rangle| \leq\|S\|_{\mathfrak{A}^{*}\left({ }^{n} E_{N}^{\times} ; F_{N}^{\times}\right)} \cdot\|T\|_{\mathfrak{A}\left({ }^{n} E_{N} ; F_{N}\right)}$. Moreover, if $S$ is diagonal, there exists a sequence $\beta$ such that $S=S_{\beta}=\sum_{j=1}^{N} \beta(j) \cdot e_{j} \otimes \cdots \otimes e_{j} \otimes e_{j}^{\times}$. Then, $\left\langle S_{\beta}, T\right\rangle=\left\langle S_{\beta}, D(T)\right\rangle$, where $D(T)=\sum_{i=1}^{N} T\left(e_{i}, \ldots, e_{i}\right)(i) \cdot e_{i}^{\times} \otimes \cdots \otimes e_{i}^{\times} \otimes e_{i}$. A direct argument through this last observation yields to the following result.

Lemma 2.5. Let $\mathfrak{A}$ be an ideal of $n$-linear operators and let $E$ and $F$ be Köthe sequence spaces. Then, $\boldsymbol{\ell}_{\boldsymbol{n}}\left(\mathfrak{A} ; E_{N}, F_{N}\right)^{\times} \stackrel{1}{=} \boldsymbol{\ell}_{\boldsymbol{n}}\left(\mathfrak{A}^{*} ; E_{N}^{\times}, F_{N}^{\times}\right)$.

As a consequence of the preceding lemma and the identity (2) we have

$$
\boldsymbol{\ell}_{\boldsymbol{n}}(\mathfrak{A} ; E, F)_{N}^{\times} \stackrel{1}{=} \boldsymbol{\ell}_{\boldsymbol{n}}\left(\mathfrak{A} ; E_{N}, F_{N}\right)^{\times} \stackrel{1}{=} \boldsymbol{\ell}_{\boldsymbol{n}}\left(\mathfrak{A}^{*} ; E_{N}^{\times}, F_{N}^{\times}\right) \stackrel{1}{=} \boldsymbol{\ell}_{\boldsymbol{n}}\left(\mathfrak{A}^{*} ; E^{\times}, F^{\times}\right)_{N} .
$$

This equality allows us to give a general result that relates the sequence space associated to an ideal with the corresponding sequence space associated to its adjoint ideal.

Proposition 2.6. Let $\mathfrak{A}$ be an ideal of n-linear operators and let $E$ and $F$ be Köthe sequence spaces. Then, $\boldsymbol{\ell}_{\boldsymbol{n}}(\mathfrak{A} ; E, F)^{\times} \stackrel{1}{=} \boldsymbol{\ell}_{\boldsymbol{n}}\left(\mathfrak{A}^{*} ; E^{\times}, F^{\times}\right)$.

Proof. For a sequence $\alpha$, by identity (41), we have $\left(\boldsymbol{\ell}_{\boldsymbol{n}}(\mathfrak{A} ; E, F)^{\times}\right)^{\max } \stackrel{1}{=} \boldsymbol{\ell}_{\boldsymbol{n}}\left(\mathfrak{A}^{*} ; E^{\times}, F^{\times}\right)^{\text {max }}$. Since a Köthe dual and an adjoint ideal are maximal, Proposition 2.2 gives the result.

Finally, as a consequence of the Propositions 2.6 and 2.2 and the fact that $\mathfrak{A}^{* *} \stackrel{1}{=} \mathfrak{A}^{\text {max }}$, we obtain the following equalities:

$$
\boldsymbol{\ell}_{\boldsymbol{n}}\left(\mathfrak{A}^{*} ; E^{\times}, F^{\times}\right)^{\times} \stackrel{1}{=} \boldsymbol{\ell}_{\boldsymbol{n}}\left(\mathfrak{A}^{* *} ; E^{\times \times}, F^{\times \times}\right) \stackrel{1}{=} \boldsymbol{\ell}_{\boldsymbol{n}}\left(\mathfrak{A}^{\max } ; E, F^{\times \times}\right) .
$$

In particular, if $\mathfrak{A}$ and $F$ are maximal, $\boldsymbol{\ell}_{\boldsymbol{n}}(\mathfrak{A} ; E, F) \stackrel{1}{=} \boldsymbol{\ell}_{\boldsymbol{n}}\left(\mathfrak{A}^{*} ; E^{\times}, F^{\times}\right)^{\times}$. 


\section{Some Applications: Multipliers and Lorentz SEQUenCe SPACES}

An example of a sequence space associated to a set of operators is the space of multipliers from $E$ into $F, \mathcal{M}(E, F)$ [13], which is defined, in our notation, as

$$
\mathcal{M}(E ; F)=\boldsymbol{\ell}_{\mathbf{1}}(\mathcal{L} ; E, F)
$$

We begin by showing that our sequence space associated to a multilinear ideal can be seen inside a suitable space of multipliers.

Proposition 3.1. Let $\mathfrak{A}$ be an ideal of n-linear operators and let $E$ and $F$ be Köthe sequence spaces. Then, $\boldsymbol{\ell}_{\boldsymbol{n}}(\mathfrak{A} ; E, F) \stackrel{\leq 1}{\hookrightarrow} \mathcal{M}\left(F^{\times}, \boldsymbol{\ell}_{\boldsymbol{n}}(\mathfrak{A} ; E)\right)$.

Proof. Let $\alpha \in \boldsymbol{\ell}_{\boldsymbol{n}}(\mathfrak{A} ; E, F)$ and let $\beta \in F^{\times}$. Consider $\varphi_{\beta} \in F^{\prime}$ given by $\varphi_{\beta}(x)=$ $\sum_{k \in \mathbb{N}} \beta(k) \cdot x(k)$. If we compose $\varphi_{\beta}$ with $T_{\alpha}$, we obtain $\phi_{\alpha \cdot \beta}$ the diagonal $n$-linear form associated to $\alpha \cdot \beta$. Then, by the ideal property, $\phi_{\alpha \cdot \beta}$ belongs to $\mathfrak{A}\left({ }^{n} E\right)$ and

$$
\left\|\phi_{\alpha \cdot \beta}\right\|_{\mathfrak{A}\left({ }^{n} E\right)} \leq\left\|\varphi_{\beta}\right\|_{F^{\prime}} \cdot\left\|T_{\alpha}\right\|_{\mathfrak{A}\left({ }^{n} E ; F\right)}=\|\beta\|_{F^{\times}} \cdot\|\alpha\|_{\ell_{n}(\mathfrak{A} ; E, F)} .
$$

In consequence, $\alpha$ belongs to $\mathcal{M}\left(F^{\times}, \boldsymbol{\ell}_{\boldsymbol{n}}(\mathfrak{A} ; E)\right)$ and $\|\alpha\|_{\mathcal{M}\left(F^{\times}, \ell_{n}(\mathfrak{A} ; E)\right)} \leq\|\alpha\|_{\ell_{n}(\mathfrak{A} ; E, F)}$.

In general, the inclusion given in Proposition 3.1 is not an equality. For example, in [9] it is proved that $\ell_{\boldsymbol{n}}\left(\mathcal{E} ; \ell_{\frac{3}{2}}, \ell_{4}\right)=\ell_{4} \neq \mathcal{M}\left(\ell_{4}^{\times}, \ell_{n}\left(\mathcal{E} ; \ell_{\frac{3}{2}}\right)\right)=\mathcal{M}\left(\ell_{\frac{4}{3}}, \ell_{\frac{3}{2}}\right)=\ell_{\infty}$, where $\mathcal{E}$ is the ideal of extendible multilinear operators. Another example from the same article is the following: $\ell_{\boldsymbol{n}}\left(\mathcal{I} ; \ell_{1}, \ell_{q}\right)=\ell_{q} \neq \mathcal{M}\left(\ell_{q}^{\times}, \ell_{\boldsymbol{n}}\left(\mathcal{I} ; \ell_{1}\right)\right)=\mathcal{M}\left(\ell_{q}^{\times}, \ell_{\infty}\right)=\ell_{\infty}$.

However, for the very particular case of the ideal of continuous multilinear operators the (isometric) equality holds when the target set is a Köthe dual.

Proposition 3.2. For Köthe sequence spaces $E$ and $F$ we have that

$$
\boldsymbol{\ell}_{\boldsymbol{n}}\left(\mathcal{L} ; E, F^{\times}\right) \stackrel{1}{=} \mathcal{M}\left(F, \boldsymbol{\ell}_{\boldsymbol{n}}(\mathcal{L} ; E)\right) .
$$

In particular, if $F$ is maximal, $\boldsymbol{\ell}_{\boldsymbol{n}}(\mathcal{L} ; E, F) \stackrel{1}{=} \mathcal{M}\left(F^{\times}, \boldsymbol{\ell}_{\boldsymbol{n}}(\mathcal{L} ; E)\right)$.

Proof. By Proposition $3.1, \boldsymbol{\ell}_{\boldsymbol{n}}\left(\mathcal{L} ; E, F^{\times}\right) \stackrel{\leq 1}{\longrightarrow} \mathcal{M}\left(F^{\times \times}, \boldsymbol{\ell}_{\boldsymbol{n}}(\mathcal{L} ; E)\right) \stackrel{\leq 1}{\longrightarrow} \mathcal{M}\left(F, \boldsymbol{\ell}_{\boldsymbol{n}}(\mathcal{L} ; E)\right)$. Conversely, let $\alpha \in \mathcal{M}\left(F, \boldsymbol{\ell}_{\boldsymbol{n}}(\mathcal{L}, E)\right)$. For any $x_{1}, \ldots, x_{n} \in E$ and $\beta \in F$, we have

$$
\left|\sum_{k \in \mathbb{N}} \alpha(k) \cdot x_{1}(k) \cdots x_{n}(k) \cdot \beta(k)\right|=\left|\phi_{\alpha \cdot \beta}\left(x_{1}, \ldots x_{n}\right)\right| \leq\left\|\phi_{\alpha \cdot \beta}\right\|_{\mathcal{L}\left({ }^{n} E\right)} \cdot\left\|x_{1}\right\|_{E} \cdots\left\|x_{n}\right\|_{E} .
$$

So, $T_{\alpha} \in \mathcal{L}\left({ }^{n} E ; F^{\times}\right)$and

$$
\|\alpha\|_{\ell_{\boldsymbol{n}}\left(\mathcal{L} ; E, F^{\times}\right)}=\left\|T_{\alpha}\right\|_{\mathcal{L}\left({ }^{n} E ; F^{\times}\right)} \leq \sup _{\beta \in B_{F}}\left\|\phi_{\alpha \cdot \beta}\right\|_{\mathcal{L}\left({ }^{n} E\right)}=\sup _{\beta \in B_{F}}\|\alpha \cdot \beta\|_{\ell_{n}(\mathcal{L} ; E)}=\|\alpha\|_{\mathcal{M}\left(F, \ell_{n}(\mathcal{L} ; E)\right)}
$$

Last, if $F$ is maximal, then $\boldsymbol{\ell}_{\boldsymbol{n}}(\mathcal{L} ; E, F) \stackrel{1}{=} \boldsymbol{\ell}_{\boldsymbol{n}}\left(\mathcal{L} ; E, F^{\times \times}\right) \stackrel{1}{=} \mathcal{M}\left(F^{\times}, \boldsymbol{\ell}_{\boldsymbol{n}}(\mathcal{L} ; E)\right)$.

Note that if $F$ is not maximal, the equality $\boldsymbol{\ell}_{\boldsymbol{n}}(\mathcal{L} ; E, F) \stackrel{1}{=} \mathcal{M}\left(F^{\times}, \boldsymbol{\ell}_{\boldsymbol{n}}(\mathcal{L} ; E)\right)$ might not be true. For instance, take $E=\ell_{\infty}$ and $F=c_{0}$, then $\ell_{\boldsymbol{n}}\left(\mathcal{L} ; \ell_{\infty}, c_{0}\right)=c_{0} \neq$ $\mathcal{M}\left(\ell_{1} ; \ell_{n}\left(\mathcal{L} ; \ell_{\infty}\right)\right)=\mathcal{M}\left(\ell_{1}, \ell_{1}\right)=\ell_{\infty}$

Corollary 3.3. Let $E$ and $F$ be Köthe sequence spaces. Then, 


$$
\boldsymbol{\ell}_{\boldsymbol{n}}\left(\mathcal{I} ; E, F^{\times}\right) \stackrel{1}{=} \boldsymbol{\ell}_{\mathbf{1}}\left(\mathcal{I} ; F, \boldsymbol{\ell}_{\boldsymbol{n}}(\mathcal{I} ; E)\right)
$$

In particular, if $F$ is maximal, $\boldsymbol{\ell}_{\boldsymbol{n}}(\mathcal{I} ; E, F) \stackrel{1}{=} \boldsymbol{\ell}_{\mathbf{1}}\left(\mathcal{I} ; F^{\times}, \boldsymbol{\ell}_{\boldsymbol{n}}(\mathcal{I} ; E)\right)$.

Proof. Being $\mathcal{I}^{*} \stackrel{1}{=} \mathcal{L}$ and $\mathcal{L}^{*} \stackrel{1}{=} \mathcal{I}$, we have

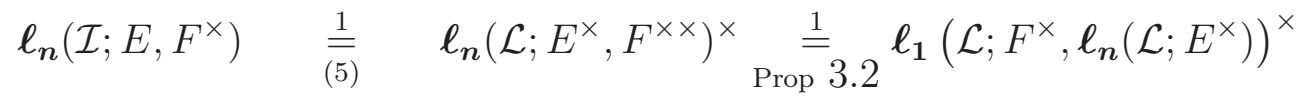

$$
\begin{aligned}
& \underset{\text { Prop }}{\stackrel{1}{=} 2.6} \ell_{1}\left(\mathcal{I} ; F^{\times \times}, \ell_{n}\left(\mathcal{L} ; E^{\times}\right)^{\times}\right) \underset{\text { Prop } 2.2}{\stackrel{1}{=}} \ell_{1}\left(\mathcal{I} ; F, \ell_{n}\left(\mathcal{L} ; E^{\times}\right)^{\times}\right) \\
& \stackrel{1}{=} \boldsymbol{\ell}_{\mathbf{1}}\left(\mathcal{I} ; F, \boldsymbol{\ell}_{\boldsymbol{n}}\left(\mathcal{I} ; E^{\times \times}\right)\right) \stackrel{1}{=} \boldsymbol{\ell}_{\mathbf{1}}\left(\mathcal{I} ; F, \boldsymbol{\ell}_{\boldsymbol{n}}(\mathcal{I} ; E)\right) \text {, }
\end{aligned}
$$

where the last two equalities hold by [8, Prop 5.5, Prop 5.6].

Recall the definition of powers of sequence spaces. Let $E$ be a Köthe sequence space and $0<r<\infty$ such that $\mathbf{M}^{(\max (1, r))}(E)=1$. Then, $E^{r}:=\left\{x \in \ell_{\infty}:|x|^{1 / r}=\left(|x(k)|^{1 / r}\right)_{k \in \mathbb{N}} \in\right.$ $E\}$ endowed with the norm $\|x\|_{E^{r}}:=\left\||x|^{1 / r}\right\|_{E}^{r}$ results a Köthe sequence space which is $\frac{1}{\min (1, r)}$-convex. And, the sequence space $E^{r}$ is maximal if $E$ is maximal.

Observe that since $E$ is normal, we can use $x^{1 / r}$ instead of $|x|^{1 / r}$ in the definition of $E^{r}$ and its norm.

Remark 3.4. Whenever $x_{1}, \ldots, x_{n} \in B_{E}$, then $\left(x_{1} \cdots x_{n}\right)^{1 / n} \in B_{E}$. Indeed, we have that $\left\|\left(\left|x_{1} \cdots x_{n}\right|\right)^{1 / n}\right\|_{E} \leq\left\|\frac{\left|x_{1}\right|+\cdots+\left|x_{n}\right|}{n}\right\|_{E} \leq \frac{\left\|x_{1}\right\|_{E}+\cdots+\left\|x_{n}\right\|_{E}}{n} \leq 1$. In particular, for an $n$-convex Köthe sequence space $E$, if $x_{1}, \ldots, x_{n} \in \stackrel{n}{B_{E}}$, then $x_{1} \cdots x_{n} \in B_{E^{n}}$.

In the case that $E$ is $n$-convex, there is an alternative description of $\boldsymbol{\ell}_{\boldsymbol{n}}(\mathcal{L} ; E, F)$ as a space of multipliers:

Proposition 3.5. Let $E$ and $F$ be an Köthe sequence spaces such that $E$ is n-convex with $\mathbf{M}^{(n)}(E)=1$. Then, $\boldsymbol{\ell}_{\boldsymbol{n}}(\mathcal{L} ; E, F) \stackrel{1}{=} \mathcal{M}\left(E^{n}, F\right)$.

Proof. Let $\alpha \in \boldsymbol{\ell}_{\boldsymbol{n}}(\mathcal{L} ; E, F)$ and take $x \in E^{n}$. Then $x^{1 / n} \in E$ and $T_{\alpha}\left(x^{1 / n}, \ldots, x^{1 / n}\right)=$ $\alpha \cdot x^{1 / n} \cdots x^{1 / n}=\alpha \cdot x \in F$. Thus, $\alpha \in \mathcal{M}\left(E^{n}, F\right)$ and

$$
\begin{aligned}
\|\alpha\|_{\mathcal{M}\left(E^{n}, F\right)} & =\sup _{x \in B_{E^{n}}}\|\alpha \cdot x\|_{F}=\sup _{x \in B_{E^{n}}}\left\|T_{\alpha}\left(x^{1 / n}, \ldots, x^{1 / n}\right)\right\|_{F} \leq \sup _{x \in B_{E^{n}}}\left\|T_{\alpha}\right\|_{\mathcal{L}\left({ }^{n} E ; F\right)} \cdot\left\|x^{1 / n}\right\|_{E}^{n} \\
& =\sup _{x \in B_{E^{n}}}\|\alpha\|_{\ell_{n}(\mathcal{L} ; E, F)} \cdot\|x\|_{E^{n}}=\|\alpha\|_{\ell_{n}(\mathcal{L} ; E, F)} .
\end{aligned}
$$

Conversely, let $\alpha \in \mathcal{M}\left(E^{n}, F\right)$. Then, $T_{\alpha}\left(x_{1}, \ldots, x_{n}\right)=\alpha \cdot x_{1} \cdots x_{n} \in F$, for all $x_{1}, \ldots, x_{n} \in E$. In consequence, $T_{\alpha}$ is well defined from $E \times \cdots \times E$ to $F$ and

$$
\left\|T_{\alpha}\right\|_{\mathcal{L}\left({ }^{n} E ; F\right)}=\sup _{x_{i} \in B_{E}}\left\|\alpha \cdot x_{1} \cdots x_{n}\right\|_{F} \leq \sup _{x \in B_{E^{n}}}\|\alpha \cdot x\|_{F}=\|\alpha\|_{\mathcal{M}\left(E^{n}, F\right)} .
$$

As a direct consequence of Propositions 3.2 and 3.5 we have: 
Corollary 3.6. Let $E$ and $F$ be an Köthe sequence spaces such that $E$ is n-convex with $\mathbf{M}^{(n)}(E)=1$. Then, $\mathcal{M}\left(E^{n}, F^{\times}\right) \stackrel{1}{=} \mathcal{M}\left(F, \boldsymbol{\ell}_{\boldsymbol{n}}(\mathcal{L} ; E)\right)$.

Recall the definition of Lorentz sequence spaces. For each element $x \in E$ its decreasing rearrangement $\left(x^{\star}(k)\right)_{k \in \mathbb{N}}$ is given by $x^{\star}(k):=\inf \left\{\sup _{j \in \mathbb{N} \backslash J}|x(j)|: J \subseteq \mathbb{N}, \operatorname{card}(J)<k\right\}$. Let $(w(k))_{k=1}^{\infty}$ be a decreasing sequence of positive numbers with $w(1)=1, w(k)$ tends to zero and $\sum_{k=1}^{\infty} w(k)=\infty$ and let $1 \leq p<\infty$. Then the corresponding Lorentz sequence space, denoted by $d(w, p)$ is defined as the set of all sequences $(x(k))_{k}$ such that

$$
\|x\|=\sup _{\sigma \in \Sigma_{\mathbb{N}}}\left(\sum_{k=1}^{\infty}|x(\sigma(k))|^{p} \cdot w(k)\right)^{1 / p}=\left(\sum_{k=1}^{\infty}\left|x^{\star}(k)\right|^{p} \cdot w(k)\right)^{1 / p}<\infty,
$$

where $\Sigma_{\mathbb{N}}$ denotes the group of permutations of the natural numbers.

The sequence $w$ is said to be $\alpha$-regular $(0<\alpha<\infty)$ if $w(k)^{\alpha} \asymp \frac{1}{k} \sum_{j=1}^{k} w(j)^{\alpha}$ and regular if it is $\alpha$-regular for some $\alpha$. In [37] it can be found that the Köthe sequence space $d(w, p)$ is $r$-convex (with $\mathbf{M}^{(r)}(d(w, p))=1$ ) whenever $1 \leq r \leq p$. In [21] and [28] a description of $d(w, p)^{\prime}$, the dual of $d(w, p)$, is given. In the case that $w$ is regular, an easier description of $d(w, p)^{\prime}$ with $p>1$ is given in [1, 38]. Let us recall also that, given a strictly positive, increasing sequence $\Psi$ such that $\Psi(0)=0$, the associated Marcinkiewicz sequence space $m_{\Psi}$ (see [23, Definition 4.1], or [11, 22]) consists of all sequences $(x(k))_{k}$ such that $\|x\|_{m_{\Psi}}=\sup _{N} \frac{\sum_{k=1}^{N} x^{\star}(k)}{\Psi(N)}<\infty$.

The results of the previous section combined with the scalar-valued case for Lorentz spaces studied in [8, Section 5] allow us to give a description of diagonal multilinear mappings from Lorentz sequence spaces (or their duals).

Proposition 3.2 along with [8] produce

$$
\boldsymbol{\ell}_{\boldsymbol{n}}\left(\mathcal{L} ; d(w, p), F^{\times}\right) \stackrel{1}{=} \mathcal{M}\left(F, \boldsymbol{\ell}_{\boldsymbol{n}}(\mathcal{L} ; d(w, p))\right) \stackrel{1}{=} \begin{cases}\mathcal{M}\left(F, d(w, p / n)^{\times}\right) & \text {if } n \leq p ; \\ \mathcal{M}\left(F, m_{\Psi}\right) & \text { if } n>p\end{cases}
$$

where $\Psi(N)=\left(\sum_{k=1}^{N} w(k)\right)^{n / p}$. Moreover, $\boldsymbol{\ell}_{\boldsymbol{n}}\left(\mathcal{L} ; d(w, p), F^{\times}\right)=\mathcal{M}\left(F, \ell_{\infty}\right)=\ell_{\infty}$ if $n>p$ and $w$ is $\frac{n}{n-p}$-regular. For $n \leq p$, since $d(w, p)$ is $n$-convex with $\mathbf{M}^{(n)}(d(w, p))=1$, Proposition 3.5 gives an alternative description: $\boldsymbol{\ell}_{\boldsymbol{n}}(\mathcal{L} ; d(w, p), F)=\mathcal{M}\left(d(w, p)^{n}, F\right)=$ $\mathcal{M}(d(w, p / n), F)$. Proposition 3.2 combined with some results of [8], also imply

$$
\boldsymbol{\ell}_{\boldsymbol{n}}\left(\mathcal{L} ; d(w, p)^{\times}, F^{\times}\right) \stackrel{1}{=} \mathcal{M}\left(F, \boldsymbol{\ell}_{\boldsymbol{n}}\left(\mathcal{L} ; d(w, p)^{\times}\right)\right) \stackrel{1}{=} \begin{cases}\mathcal{M}\left(F, \ell_{\infty}\right)=\ell_{\infty} & \text { if } n^{\prime} \leq p ; \\ \mathcal{M}\left(F, d\left(w^{\frac{n^{\prime}}{n^{\prime}-p}}, \frac{p^{\prime}}{p^{\prime}-n}\right)\right) & \text { if } 1<p<n^{\prime} \\ \mathcal{M}\left(F, d\left(w^{n}, 1\right)\right) & \text { if } p=1\end{cases}
$$

To complete this description it remains to calculate the space of multipliers from $F$ to a Lorentz sequence space. We can give an explicit characterization when $F=\ell_{q}$. We affirm 
that

$$
\mathcal{M}\left(\ell_{q}, d(w, p)\right)=\left\{\begin{array}{cl}
d\left(w^{\frac{q}{q-p}}, \frac{p q}{q-p}\right) & \text { si } p<q ; \\
\ell_{\infty} & \text { si } p \geq q .
\end{array}\right.
$$

Indeed, when $p \geq q$, the equality is clear from the inclusions $\ell_{q} \subseteq \ell_{p} \subseteq d(w, p)$.

When $p<q$,

$$
\begin{aligned}
\|\alpha\|_{\mathcal{M}\left(\ell_{q}, d(w, p)\right)} & =\sup _{\beta \in B_{\ell_{q}}}\|\alpha \cdot \beta\|_{d(w, p)}=\sup _{\beta \in B_{\ell_{q}}} \sup _{\sigma \in \Sigma_{\mathbb{N}}}\left(\sum_{k \in \mathbb{N}}\left|\alpha_{\sigma}(k)\right|^{p} \cdot\left|\beta_{\sigma}(k)\right|^{p} \cdot w(k)\right)^{1 / p} \\
& =\sup _{\sigma \in \Sigma_{\mathbb{N}}} \sup _{\gamma \in B_{\frac{q}{p}}}\left(\sum_{k \in \mathbb{N}}\left|\alpha_{\sigma}(k)\right|^{p} \cdot|\gamma(k)| \cdot w(k)\right)^{1 / p}=\sup _{\sigma \in \Sigma_{\mathbb{N}}}\left(\left\|\alpha_{\sigma}^{p} \cdot w\right\|_{\left(\frac{q}{p}\right)^{\prime}}\right)^{1 / p} \\
& =\sup _{\sigma \in \Sigma_{\mathbb{N}}}\left(\sum_{k \in \mathbb{N}}\left|\alpha_{\sigma}(k)\right|^{p\left(\frac{q}{p}\right)^{\prime}} \cdot w(k)^{\left(\frac{q}{p}\right)^{\prime}}\right)^{\frac{1}{p\left(\frac{q}{p}\right)^{\prime}}}=\|\alpha\|_{d\left(w^{\frac{q}{q-p}}, \frac{p q}{q-p}\right)} .
\end{aligned}
$$

We can obtain, applying Theorem 2.6 and taking into account that $\mathcal{I}^{*}=\mathcal{L}$, similar results for the ideal of integral multilinear operators.

\section{4. (E,P)-SUMMing MULTILINEAR OPERATORS}

The classical notion of $(q, p)$-summing operator has a natural extension by changing the index $q$ (which refers to the space $\ell_{q}$ ) by any other Köthe sequence space $E$ containing $\ell_{p}$. This yields the concept of $(E, p)$-summing linear mapping. This class, denoted by $\Pi_{(E, p)}$, was studied in [13] where typical results about $(q, p)$-summing operators are extended to the case of $(E, p)$-summing linear mappings by means of the space of multipliers. Here, we propose an $n$-linear version of that program.

Along this section we consider $1 \leq p<\infty$. If $\ell_{p} \hookrightarrow E$, we denote by $c_{p}^{E}=\left\|i: \ell_{p} \hookrightarrow E\right\|$ the norm of the natural inclusion map. We need to recall also that for a Köthe sequence space $E$, the $1 / n$-convexification, $E^{1 / n}$, is always well defined and it is an $n$-convex Köthe sequence space with $\mathbf{M}^{(n)}\left(E^{1 / n}\right)=1$. Now, we can proceed to the definition.

Let $E$ be a Köthe sequence space such that $\ell_{p} \hookrightarrow E^{1 / n}$ and let $X_{1}, \ldots, X_{n}, Y$ be Banach spaces. An $n$-linear operator $T \in \mathcal{L}\left(X_{1}, \ldots, X_{n} ; Y\right)$ is called $(E, p)$-summing if there exists $C>0$ such that for every finite sequences $x_{1}=\left(x_{1, i}\right)_{i=1}^{m} \subseteq X_{1}, \ldots, x_{n}=\left(x_{n, i}\right)_{i=1}^{m} \subseteq X_{n}$ it holds

$$
\left\|\left(\left\|T\left(x_{1, i}, \ldots, x_{n, i}\right)\right\|_{Y}\right)_{i=1}^{m}\right\|_{E} \leq C \cdot c_{p}^{E^{1 / n}} \cdot w_{p}\left(x_{1}\right) \cdots w_{p}\left(x_{n}\right)
$$

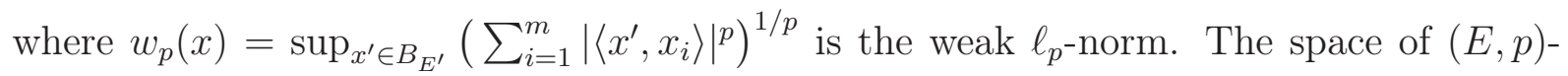
summing $n$-linear operators from $X_{1} \times \cdots \times X_{n}$ to $Y$ is denoted by $\Pi_{(E, p)}\left(X_{1}, \ldots, X_{n} ; Y\right)$. It is a Banach space endowed with the norm $\pi_{(E, p)}(T)=\inf \{C>0: T$ verifies (6) $\}$. Moreover, it is easy to see that $\Pi_{(E, p)}$ is a Banach ideal of $n$-linear operators (always under the condition $\left.\ell_{p} \hookrightarrow E^{1 / n}\right)$. 
When $E=\ell_{q}$ and $p \leq n q$, the ideal $\Pi_{(E, p)}$ is the class of $(q, p)$-summing $n$-linear mappings $\Pi_{(q, p)}$ introduced and studied by [30]. For $p=n q,(q, p)$-summing $n$-linear mappings are the so called $p$-dominated $n$-linear mappings $\mathcal{D}_{p}$ [30, 39, 31]. In the case $n=$ 1 , the class $\Pi_{(E, p)}$ is the usual ideal of $(E, p)$-summing linear operators mentioned above. When $E=\ell_{p}$ this is just the classical ideal of absolutely $p$-summing linear mappings, $\Pi_{p}$, with $\pi_{p}(\cdot)$ as the usual notation for its norm.

In the sequel we present $n$-linear versions of some results in [13 along with a relationship between the sequence space associated to the ideal $\Pi_{(E, p)}$ and a linear relative.

We begin by the $n$-linear version of [13, Lemma 3.3]. It is a standard characterization of $(E, p)$-summability with a straightforward proof that we omit.

Lemma 4.1. Let $E$ be a Köthe sequence space such that $\ell_{p} \hookrightarrow E^{1 / n}$ and let $X_{1}, \ldots, X_{n}, Y$ be Banach spaces. For a mapping $T \in \mathcal{L}\left(X_{1}, \ldots, X_{n} ; Y\right)$ and a constant $C \geq 0$ the following are equivalent:

(1) $T \in \Pi_{(E, p)}\left(X_{1}, \ldots, X_{n} ; Y\right)$ with $\pi_{(E, p)}(T) \leq C$.

(2) $\pi_{(E, p)}\left(T \circ\left(A_{1}, \ldots, A_{n}\right)\right) \leq C$ for all $m \in \mathbb{N}$ and for all $A_{j} \in \mathcal{L}\left(\ell_{p^{\prime}}^{m} ; X_{j}\right)$ with $\left\|A_{j}\right\| \leq 1$. (Here $\ell_{p^{\prime}}^{m}$ means the space $\mathbb{C}^{m}$ with the $\ell_{p^{\prime}}$-norm, not to be confused with the $m$-power of the sequence space $\ell_{p^{\prime}}$.)

In particular, in this case,

$$
\pi_{(E, p)}(T)=\sup _{m}\left\{\pi_{(E, p)}\left(T \circ\left(A_{1}, \ldots, A_{n}\right)\right):\left\|A_{j}\right\|_{\mathcal{L}\left(\ell_{p^{\prime}}^{m} ; X_{j}\right)} \leq 1, \text { for } j=1, \ldots, n\right\} .
$$

Next lemma enumerates two simple properties about a sequence space associated to an ideal that will be needed later.

Lemma 4.2. Let $E$ be a Köthe sequence space with $\ell_{p} \hookrightarrow E^{1 / n}$.

(i) If $q$ and $r$ are such that $p<q$ and $\frac{1}{r}=\frac{1}{p}-\frac{1}{q}$, then $\ell_{q} \hookrightarrow\left[\ell_{\boldsymbol{n}}\left(\mathcal{L} ; \ell_{r}, E\right)\right]^{1 / n}$.

(ii) If $F$ and $G$ are Köthe sequence spaces and $G$ is $n$-convex with $\mathbf{M}^{(n)}(G)=1$, then $\ell_{1}\left(\Pi_{\left(E^{1 / n}, p\right)} ; F, G\right)$ is n-convex with convexity constant 1.

Proof. (i) First, note that $\alpha \in\left[\ell_{n}\left(\mathcal{L} ; \ell_{r}, E\right)\right]^{1 / n}$ if and only if $\left(\alpha \cdot x_{1}^{1 / n} \cdots x_{n}^{1 / n}\right) \in E^{1 / n}$ for all $x_{1}, \ldots, x_{n} \in \ell_{r}$. Now, for $\alpha \in \ell_{q}$ and $x_{1}, \ldots, x_{n} \in \ell_{r}$, it is clear that $x_{1}^{1 / n} \cdots x_{n}^{1 / n} \in \ell_{r}$ and so $\alpha \cdot x_{1}^{1 / n} \cdots x_{n}^{1 / n} \in \ell_{p} \hookrightarrow E^{1 / n}$.

(ii) Let $\alpha_{1}, \ldots, \alpha_{N} \in \boldsymbol{\ell}_{\mathbf{1}}\left(\Pi_{\left(E^{1 / n}, p\right)} ; F, G\right)$, we have to show that

$$
\left\|\left[\left(\sum_{k=1}^{N}\left|\alpha_{k}(j)\right|^{n}\right)^{1 / n}\right]_{j=1}^{\infty}\right\|_{\ell_{1}\left(\Pi_{\left(E^{1 / n}, p\right)} ; F, G\right)} \leq\left(\sum_{k=1}^{N}\left\|\alpha_{k}\right\|_{\ell_{1}\left(\Pi_{\left(E^{1 / n}, p\right)} ; F, G\right)}\right)^{1 / n} .
$$


Equivalently, if we call $\beta(j)=\left(\sum_{k=1}^{N}\left|\alpha_{k}(j)\right|^{n}\right)^{1 / n}$, the condition to be checked is

$$
\left\|D_{\beta}\right\|_{\Pi_{\left(E^{1 / n}, p\right)}(F ; G)} \leq\left(\sum_{k=1}^{N}\left\|D_{\alpha_{k}}\right\|_{\Pi_{\left(E^{1 / n}, p\right)}^{n}(F ; G)}\right)^{1 / n} .
$$

Now, let $x_{1}, \ldots, x_{m} \in F$. Since $G$ is $n$-convex with $\mathbf{M}^{(n)}(G)=1$ we obtain

$$
\begin{aligned}
& \left\|\left(\left\|D_{\beta}\left(x_{i}\right)\right\|_{G}\right)_{i=1}^{m}\right\|_{E^{1 / n}}=\left\|\left(\left\|\beta \cdot x_{i}\right\|_{G}\right)_{i=1}^{m}\right\|_{E^{1 / n}}=\left\|\left(\left\|\beta \cdot x_{i}\right\|_{G}^{n}\right)_{i=1}^{m}\right\|_{E}^{1 / n} \\
& =\left\|\left(\left\|\left(\sum_{k=1}^{N}\left|\alpha_{k} \cdot x_{i}\right|^{n}\right)^{1 / n}\right\|_{G}^{n}\right)_{i=1}^{m}\right\|_{E}^{1 / n} \leq\left\|\left(\sum_{k=1}^{N}\left\|\alpha_{k} \cdot x_{i}\right\|_{G}^{n}\right)_{i=1}^{m}\right\|_{E}^{1 / n} \\
& \text { (by Minkowski) } \leq\left(\sum_{k=1}^{N}\left\|\left(\left\|D_{\alpha_{k}}\left(x_{i}\right)\right\|_{G}^{n}\right)_{i=1}^{m}\right\|_{E}\right)^{1 / n}=\left(\sum_{k=1}^{N}\left\|\left(\left\|D_{\alpha_{k}}\left(x_{i}\right)\right\|_{G}\right)_{i=1}^{m}\right\|_{E^{1 / n}}^{n}\right)^{1 / n} \\
& \leq\left(\sum_{k=1}^{N}\left(\left\|D_{\alpha_{k}}\right\|_{\Pi_{\left(E^{1 / n}, p\right)}} \cdot w_{p}\left(x_{i}\right)\right)^{n}\right)^{\frac{1}{n}}=\left(\sum_{k=1}^{N}\left\|D_{\alpha_{k}}\right\|_{\Pi_{\left(E^{1 / n}, p\right)}}\right)^{\frac{1}{n}} \cdot w_{p}(x) .
\end{aligned}
$$

Then, $\left\|D_{\beta}\right\|_{\Pi_{\left(E^{1 / n}, p\right)}(F ; G)} \leq\left(\sum_{k=1}^{N}\left\|D_{\alpha_{k}}\right\|_{\Pi_{\left(E^{1 / n}, p\right)}^{n}(F ; G)}\right)^{1 / n}$.

Note that under the assumptions of item (i) of lemma above the class $\prod_{\left(\ell_{n}\left(\mathcal{L} ; \ell_{r}, E\right), q\right)}$ for $n$-linear operators is well defined.

Remark 4.3. In Remark 3.4 we showed that whenever $\left\|x_{1}\right\|_{E}=\cdots=\left\|x_{n}\right\|_{E}=1$, then $\left\|\left(x_{1} \cdots x_{n}\right)^{1 / n}\right\|_{E}^{n} \leq 1$. Thus, for every $x_{1}, \ldots, x_{n} \in E$ we have $\left\|\left(x_{1} \cdots x_{n}\right)^{1 / n}\right\|_{E}^{n} \leq$ $\left\|x_{1}\right\|_{E} \cdots\left\|x_{n}\right\|_{E}$. Hence, if the space $E$ is n-convex, $\left\|x_{1} \cdots x_{n}\right\|_{E^{n}} \leq\left\|x_{1}\right\|_{E} \cdots\left\|x_{n}\right\|_{E}$. In particular, it holds that $\left\|x_{1} \cdots x_{n}\right\|_{E} \leq\left\|x_{1}\right\|_{E^{1 / n}} \cdots\left\|x_{n}\right\|_{E^{1 / n}}$, for all $x_{1}, \ldots, x_{n} \in E^{1 / n}$.

Now we present in the next theorem two composition results about $(E, p)$-summing $n$-linear mappings. Since the statement involve both linear and $n$-linear ideals to avoid confusion we chose to denote by $\Pi_{(E, p)}^{(n)}$ the ideal of $(E, p)$-summing $n$-linear operators. Observe that for the particular case of $E=\ell \frac{p}{n}$ both compositions are known results about $p$-dominated $n$-linear operators [31.

Theorem 4.4 (Composition theorem for $(E, p)$-summing multilinear mappings). Let $E$ be a Köthe sequence space such that $\ell_{p} \hookrightarrow E^{1 / n}$.

(i) If $q$ and $r$ are such that $p<q$ and $\frac{1}{r}=\frac{1}{p}-\frac{1}{q}$, then

$$
\Pi_{\left(\ell_{n}\left(\mathcal{L} ; \ell_{r}, E\right), q\right)}^{(n)} \circ\left(\Pi_{r}, \ldots, \Pi_{r}\right) \subseteq \Pi_{(E, p)}^{(n)} .
$$

Moreover, $\pi_{(E, p)}^{(n)}\left(T \circ\left(A_{1}, \ldots, A_{n}\right)\right) \leq \pi_{\left(\ell_{n}\left(\mathcal{L} ; \ell_{r}, E\right), q\right)}^{(n)}(T) \cdot \pi_{r}\left(A_{1}\right) \cdots \pi_{r}\left(A_{n}\right)$, for $T$ belonging to $\Pi_{\left(\ell_{n}\left(\mathcal{L} ; \ell_{r}, E\right), q\right)}^{(n)}$ and $A_{1}, \ldots, A_{n}$ belonging to $\Pi_{r}$. 
(ii) It holds

$$
\mathcal{L}^{(n)} \circ\left(\Pi_{\left(E^{1 / n}, p\right)}, \ldots, \Pi_{\left(E^{1 / n}, p\right)}\right) \subseteq \Pi_{(E, p)}^{(n)} .
$$

Moreover, $\pi_{(E, p)}^{(n)}\left(T \circ\left(A_{1}, \ldots, A_{n}\right)\right) \leq\|T\| \cdot \pi_{\left(E^{1 / n}, p\right)}\left(A_{1}\right) \cdots \pi_{\left(E^{1 / n}, p\right)}\left(A_{n}\right)$ for $T$ an $n$-linear operator and $A_{1}, \ldots, A_{n}$ belonging to $\Pi_{\left(E^{1 / n}, p\right)}$.

Proof. (i) This is an $n$-linear version of [13, Lemma 3.5]. The proof is similar so we omit it.

(ii) Let $x_{1}=\left(x_{1, i}\right)_{i=1}^{m} \subseteq X_{1}, \ldots, x_{n}=\left(x_{n, i}\right)_{i=1}^{m} \subseteq X_{n}$. By the normal property of $E$ and by Remark 4.3, we have

$$
\begin{gathered}
\left\|\left(\left\|T\left(A_{1}\left(x_{1, i}\right), \ldots, A_{n}\left(x_{n, i}\right)\right)\right\|_{Y}\right)_{i=1}^{m}\right\|_{E} \leq\left\|\left(\|T\| \cdot\left\|A_{1}\left(x_{1, i}\right)\right\|_{Y_{1}} \cdots\left\|A_{n}\left(x_{n, i}\right)\right\|_{Y_{n}}\right)_{i=1}^{m}\right\|_{E} \\
=\|T\| \cdot\left\|\left(\left\|A_{1}\left(x_{1, i}\right)\right\|_{Y_{1}} \cdots\left\|A_{n}\left(x_{n, i}\right)\right\|_{Y_{n}}\right)_{i=1}^{m}\right\|_{E} \\
\leq\|T\| \cdot\left\|\left(\left\|A_{1}\left(x_{1, i}\right)\right\|_{Y_{1}}\right)_{i 1}^{m}\right\|_{E^{1 / n}} \cdots\left\|\left(\left\|A_{n}\left(x_{n, i}\right)\right\|_{Y_{n}}\right)_{i=1}^{m}\right\|_{E^{1 / n}} \\
\leq\|T\| \cdot \pi_{\left(E^{1 / n}, p\right)}\left(A_{1}\right) \cdot w_{p}\left(x_{1}\right) \cdots \pi_{\left(E^{1 / n}, p\right)}\left(A_{n}\right) \cdot w_{p}\left(x_{n}\right)
\end{gathered}
$$

The next proposition shows that sequence space associated to $(E, p)$-summing $n$-linear operators can be seen as the $n$-convexification of a sequence space associated to $\left(E^{1 / n}, p\right)$ summing linear mappings. This identification extend an analogous result for scalar-valued $p$-dominated $n$-linear mappings proved in [7] (see explanation below).

Proposition 4.5. Let $E, F$ and $G$ be Köthe sequence spaces such that $\ell_{p} \hookrightarrow E^{1 / n}$. Then

$$
\boldsymbol{\ell}_{\boldsymbol{n}}\left(\Pi_{(E, p)} ; F, G\right) \stackrel{1}{=}\left[\ell_{1}\left(\Pi_{\left(E^{1 / n}, p\right)} ; F, G^{1 / n}\right)\right]^{n} .
$$

Proof. Note first that $\alpha \in\left[\ell_{1}\left(\Pi_{\left(E^{1 / n}, p\right)} ; F, G^{1 / n}\right)\right]^{n}$ if and only if the diagonal linear operator $D_{\alpha^{1 / n}} \in \Pi_{\left(E^{1 / n}, p\right)}\left(F ; G^{1 / n}\right)$. Let $\alpha \in \boldsymbol{\ell}_{\boldsymbol{n}}\left(\Pi_{(E, p)} ; F, G\right)$ and take $x_{1}, \ldots, x_{m} \in F$, then

$$
\begin{aligned}
& \left\|\left(\left\|D_{\alpha^{1 / n}}\left(x_{i}\right)\right\|_{G^{1 / n}}\right)_{i=1}^{m}\right\|_{E^{1 / n}}=\left\|\left(\left\|\left(\alpha^{1 / n} \cdot x_{i}\right)^{n}\right\|_{G}^{1 / n}\right)_{i=1}^{m}\right\|_{E^{1 / n}} \\
& =\left\|\left(\left\|T_{\alpha}\left(x_{i}, \ldots, x_{i}\right)\right\|_{G}^{1 / n}\right)_{i=1}^{m}\right\|_{E^{1 / n}}=\left\|\left(\left\|T_{\alpha}\left(x_{i}, \ldots, x_{i}\right)\right\|_{G}\right)_{i=1}^{m}\right\|_{E}^{1 / n} \\
& \leq\left(\left\|T_{\alpha}\right\|_{\Pi_{(E, p)}} \cdot w_{p}(x)^{n}\right)^{1 / n}=\|\alpha\|_{\ell_{n}\left(\Pi_{(E, p)} ; F, G\right)}^{1 / n} \cdot w_{p}(x) .
\end{aligned}
$$

Then, $\alpha \in\left[\ell_{1}\left(\Pi_{\left(E^{1 / n}, p\right)} ; F, G^{1 / n}\right)\right]^{n}$ and

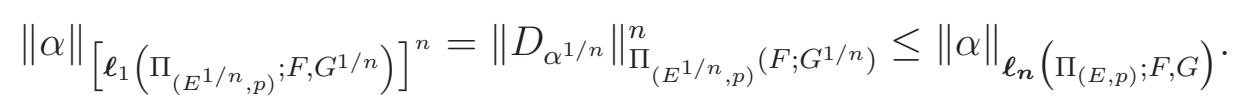


Conversely, let $\alpha \in\left[\ell_{1}\left(\Pi_{\left(E^{1 / n}, p\right)} ; F, G^{1 / n}\right)\right]^{n}$. Consider the factorization of $T_{\alpha}=\Psi \circ$ $\left(D_{\alpha^{1 / n}}, \ldots, D_{\alpha^{1 / n}}\right)$, where the operator $\Psi \in \mathcal{L}\left({ }^{n} G^{1 / n} ; G\right)$ is given by $\Psi\left(x_{1}, \ldots, x_{n}\right)=$ $x_{1} \cdots x_{n}$, and $D_{\alpha^{1 / n}} \in \Pi_{\left(E^{1 / n}, p\right)}\left(F ; G^{1 / n}\right)$. Applying Theorem $4.4(2)$, we obtain that $T_{\alpha} \in$ $\Pi_{(E, p)}\left({ }^{n} F ; G\right)$ and $\left.\pi_{(E, p)}\left(T_{\alpha}\right) \leq\|\Psi\| \cdot\left(\pi_{\left(E^{1 / n}, p\right)}\left(D_{\alpha^{1 / n}}\right)\right)^{n} \leq\|\alpha\|_{\left[\ell_{1}\left(\Pi_{\left(E^{1 / n}, p\right)} ; F, G^{1 / n}\right)\right.}\right]^{n}$.

Some comments are in order. As we have mentioned, if $p \geq n$ and $E=\ell_{\frac{p}{n}}$ then for $n$-linear mappings $\Pi_{(E, p)}$ coincides with $\mathcal{D}_{p}$ (the ideal of $p$-dominated mappings). For this particular case, the identity of the previous proposition reads as follows:

$$
\ell_{n}\left(\mathcal{D}_{p} ; F, G\right) \stackrel{1}{=}\left[\ell_{1}\left(\Pi_{p} ; F, G^{1 / n}\right)\right]^{n} .
$$

This can be seen as the vector-valued version of [7, Prop. 2.1] which, translated to our current terminology says (for $n \geq 2$ ):

$$
\ell_{n}\left(\mathcal{D}_{p} ; F\right) \stackrel{1}{=}\left[\ell_{1}\left(\Pi_{p} ; F, \ell_{n}\right)\right]^{n}
$$

Actually, that result was just for $F$ an $\ell_{p}$ space, but the same argument works for any Köthe sequence space. Moreover, it can also be proved, following analogous arguments that (when $\ell_{p} \hookrightarrow E^{1 / n}$ and $n \geq 2$ )

$$
\ell_{n}\left(\Pi_{(E, p)} ; F\right) \stackrel{1}{=}\left[\ell_{1}\left(\Pi_{\left(E^{1 / n}, p\right)} ; F, \ell_{n}\right)\right]^{n} .
$$

As an interesting consequence of these identities we derive, for every $n \geq 2$,

$$
\ell_{n}\left(\Pi_{(E, p)} ; F, \ell_{1}\right) \stackrel{1}{=} \ell_{n}\left(\Pi_{(E, p)} ; F\right) .
$$

Note that clearly this equality holds also for the ideal $\mathcal{L}$ : for $n \geq 2, \boldsymbol{\ell}_{\boldsymbol{n}}\left(\mathcal{L} ; F, \ell_{1}\right) \stackrel{1}{=}$ $\boldsymbol{\ell}_{\boldsymbol{n}}(\mathcal{L} ; F)$. However it is not true for any ideal $\mathfrak{A}$. For instance, for the ideal $\mathcal{I}$ of integral multilinear mappings we know, from identity (5) that $\boldsymbol{\ell}_{\boldsymbol{n}}\left(\mathcal{I} ; E, \ell_{1}\right) \stackrel{1}{=} \boldsymbol{\ell}_{\boldsymbol{n}}\left(\mathcal{L} ; E^{\times}, \ell_{\infty}\right)^{\times} \stackrel{1}{=} \ell_{1}$, for any Köthe sequence space $E$. But $\ell_{\boldsymbol{n}}(\mathcal{I} ; E)$ is not always equal to $\ell_{1}$. Indeed, by [7, Prop. 1.2] (see also [9]), $\ell_{n}\left(\mathcal{I} ; \ell_{p}\right) \stackrel{1}{=} \ell_{\frac{p^{\prime}}{n}}$, for $1 \leq p \leq \frac{n}{n-1}$.

Finally, we extend to the multilinear setting an Inclusion theorem for $(E, p)$-summing operators proved in [13, Lemma 3.4]. The proof is similar, so we omit it. For $E=\ell_{\frac{p}{n}}$, the first inclusion is just the usual inclusion of $p$-dominated into $q$-dominated $n$-linear operators when $p<q$. Other inclusion results about $(q, p)$-summing multilinear mappings with and without hypothesis about cotype 2 spaces in the domain can be found in [4].

Theorem 4.6 (Inclusion theorem for $(E, p)$-summing multilinear operators). Let $E$ be a Köthe sequence space such that $\ell_{p} \hookrightarrow E^{1 / n}$. If $q$ and $r$ are such that $p<q$ and $\frac{1}{r}=\frac{1}{p}-\frac{1}{q}$, then we have the following inclusion for ideals of $n$-linear mappings:

$$
\Pi_{(E, p)} \subseteq \Pi_{\left(\ell_{n}\left(\mathcal{L} ; \ell_{r}, E\right), q\right)},
$$

with $\pi_{\left(\ell_{n}\left(\mathcal{L} ; \ell_{r}, E\right), q\right)}(T) \leq c_{p}^{E^{1 / n}} \cdot\left(c_{q}^{\ell_{n}\left(\mathcal{L} ; \ell_{r}, E\right)}\right)^{-1} \cdot \pi_{(E, p)}(T)$, for all $T \in \Pi_{(E, p)}^{(n)}$. 
Moreover, if $X_{1}, \ldots, X_{n}$ have cotype 2, then for any Banach space $Y$,

$$
\Pi_{\left(\ell_{n}\left(\mathcal{L} ; \ell_{2}, E\right), 2\right)}\left(X_{1}, \ldots, X_{n} ; Y\right)=\Pi_{(E, 1)}\left(X_{1}, \ldots, X_{n} ; Y\right) .
$$

In the same spirit of the definition of $(E, p)$-summing multilinear operators and having in mind the concept of strongly $p$-summing multilinear operators [15], we introduce the class of strongly $(E, p)$-summing multilinear operators. Let $E$ be a Köthe sequence space such that $\ell_{p} \hookrightarrow E$. An $n$-linear operator $T \in \mathcal{L}\left(X_{1}, \ldots, X_{n} ; Y\right)$ is said to be strongly $(E, p)$ summing if exists $C>0$ such that for finite sequences $\left(x_{1, i}\right)_{i=1}^{m} \subseteq X_{1}, \ldots,\left(x_{n, i}\right)_{i=1}^{m} \subseteq X_{n}$, it satisfies that

$$
\left\|\left(\left\|T\left(x_{1, i}, \ldots, x_{n, i}\right)\right\|_{Y}\right)_{i=1}^{m}\right\|_{E} \leq C \cdot c_{p}^{E} \cdot \sup _{\phi \in B_{\mathcal{L}\left(X_{1}, \ldots, X_{n}\right)}}\left(\sum_{i=1}^{m}\left|\phi\left(x_{1, i}, \ldots, x_{n, i}\right)\right|^{p}\right)^{1 / p} .
$$

We note $\mathcal{S}_{(E, p)}$ the space of strongly $(E, p)$-summing multilinear operators. It is easy to see that it is an ideal of $n$-linear operators endowed with the norm

$$
S_{(E, p)}(T)=\inf \{C: T \text { verifies (마) }\} .
$$

Applying the same arguments used in [13] to prove the inclusion theorem for $(E, p)$ summing linear operators, it can be proved an analogous inclusion theorem for strongly $(E, p)$-summing multilinear operators.

Theorem 4.7. Let $E$ be a Köthe sequence space such that $\ell_{p} \hookrightarrow E$. If $\frac{n}{r}=\frac{1}{p}-\frac{1}{q}$, then

$$
\mathcal{S}_{(E, p)} \subseteq \mathcal{S}_{\left(\ell_{n}\left(\mathcal{L} ; \ell_{r}, E\right), q\right)}
$$

Moreover, if $T \in \mathcal{S}_{(E, p)}$, then $S_{\left(\ell_{n}\left(\mathcal{L} ; \ell_{r}, E\right), q\right)}(T) \leq c_{p}^{E} \cdot\left(c_{q}^{\ell_{n}\left(\mathcal{L} ; \ell_{r}, E\right)}\right)^{-1} \cdot S_{(E, p)}(T)$.

Acknowledgements. We would like to thank Daniel Carando for helpful conversations and for suggesting some of the problems developed in the article. We also want to thank the anonymous referee for useful comments that led to a better presentation of Section 4 .

\section{REFERENCES}

[1] G. D. Allen. Duals of Lorentz spaces. Pacific J. Math., 77(2):287-291, 1978.

[2] S. Aywa and J.H. Fourie. On summing multipliers and applications. J. Math. Anal. Appl., 253(1):166$186,2001$.

[3] C. Bennett and R. Sharpley. Interpolation of operators, volume 129 of Pure and Applied Mathematics. Academic Press, Inc., Boston, MA, 1988.

[4] G. Botelho, C. Michels, and D. Pellegrino. Complex interpolation and summability properties of multilinear operators. Rev. Mat. Complut., 23(1):139-161, 2010.

[5] M. Buntinas and G. Goes. Products of sequence spaces and multipliers. Rad. Mat., 3(2):287-300, 1987.

[6] E. Çalişkan and D. M. Pellegrino. On the multilinear generalizations of the concept of absolutely summing operators. Rocky Mountain J. Math., 37(4):1137-1154, 2007.

[7] D. Carando, V. Dimant, and P. Sevilla-Peris. Limit orders and multilinear forms on $l_{p}$ spaces. Publ. Res. Inst. Math. Sci., 42(2):507-522, 2006. 
[8] D. Carando, V. Dimant, and P. Sevilla-Peris. Multilinear Hölder-type inequalities on Lorentz sequence spaces. Studia Math., 195(2):127-146, 2009.

[9] D. Carando, V. Dimant, P. Sevilla-Peris, and R. Villafañe. Diagonal extendible multilinear operators between $\ell_{p}$-spaces. Rev. R. Acad. Cienc. Exactas Fís. Nat. Ser. A Math. RACSAM, 108(2):541-555, 2014.

[10] B. Carl. A remark on $p$-integral and $p$-absolutely summing operators from $\ell_{u}$ into $\ell_{v}$. Studia Math., $57(3): 257-262,1976$.

[11] Y. S. Choi and K. H. Han. Boundaries for algebras of holomorphic functions on Marcinkiewicz sequence spaces. J. Math. Anal. Appl., 323(2):1116-1133, 2006.

[12] A. Defant and K. Floret. Tensor norms and operator ideals, volume 176 of North-Holland Mathematics Studies. North-Holland Publishing Co., Amsterdam, 1993.

[13] A. Defant, M. Mastylo, and C. Michels. Summing inclusion maps between symmetric sequence spaces. Trans. Amer. Math. Soc., 354(11):4473-4492 (electronic), 2002.

[14] A. Defant, M. Mastyło, and C. Michels. Summing norms of identities between unitary ideals. Math. Z., 252(4):863-882, 2006.

[15] V. Dimant. Strongly p-summing multilinear operators. J. Math. Anal. Appl., 278(1):182-193, 2003.

[16] S. Dineen. Complex analysis on infinite-dimensional spaces. Springer Monographs in Mathematics. Springer-Verlag London, Ltd., London, 1999.

[17] K. Floret. Minimal ideals of $n$-homogeneous polynomials on Banach spaces. Results Math., 39(34):201-217, 2001.

[18] K. Floret. On ideals of $n$-homogeneous polynomials on Banach spaces. In Topological algebras with applications to differential geometry and mathematical physics (Athens, 1999), pages 19-38. Univ. Athens, Athens, 2002.

[19] K. Floret and D. García. On ideals of polynomials and multilinear mappings between Banach spaces. Arch. Math. (Basel), 81(3):300-308, 2003.

[20] K. Floret and S. Hunfeld. Ultrastability of ideals of homogeneous polynomials and multilinear mappings on Banach spaces. Proc. Amer. Math. Soc., 130(5):1425-1435 (electronic), 2002.

[21] D. J. H. Garling. A class of reflexive symmetric BK-spaces. Canad. J. Math., 21:602-608, 1969.

[22] A. Kamińska and H. J. Lee. M-ideal properties in Marcinkiewicz spaces. Comment. Math. Prace Mat., (Tomus specialis in Honorem Juliani Musielak):123-144, 2004.

[23] A. Kamińska and H. J. Lee. On uniqueness of extension of homogeneous polynomials. Houston J. Math., 32(1):227-252 (electronic), 2006.

[24] E. Karapinar. Nuclearity and multipliers between Banach spaces. Int. J. Contemp. Math. Sci., 2(2932):1601-1606, 2007.

[25] P. Kolwicz, K. Leśnik, and L. Maligranda. Pointwise multipliers of Calderón-Lozanovskiı̌ spaces. Math. Nachr., 286(8-9):876-907, 2013.

[26] P. Kolwicz, K. Leśnik, and L. Maligranda. Pointwise products of some Banach function spaces and factorization. J. Funct. Anal., 266(2):616-659, 2014.

[27] H. König. Diagonal and convolution operators as elements of operator ideals. Math. Ann., 218(2):97106,1975 .

[28] J. Lindenstrauss and L. Tzafriri. Classical Banach spaces. I. Springer-Verlag, Berlin-New York, 1977. Sequence spaces, Ergebnisse der Mathematik und ihrer Grenzgebiete, Vol. 92.

[29] J. Lindenstrauss and L. Tzafriri. Classical Banach spaces. II, volume 97 of Ergebnisse der Mathematik und ihrer Grenzgebiete [Results in Mathematics and Related Areas]. Springer-Verlag, Berlin-New York, 1979. Function spaces. 
[30] M. C. Matos. On multilinear mappings of nuclear type. Rev. Mat. Univ. Complut. Madrid, 6(1):6181, 1993.

[31] Y. Meléndez and A. Tonge. Polynomials and the Pietsch domination theorem. Math. Proc. R. Ir. Acad., 99A(2):195-212, 1999.

[32] J. Mujica. Complex analysis in Banach spaces, volume 120 of North-Holland Mathematics Studies. North-Holland Publishing Co., Amsterdam, 1986. Holomorphic functions and domains of holomorphy in finite and infinite dimensions, Notas de Matemática [Mathematical Notes], 107.

[33] D. Pellegrino and J. Santos. Absolutely summing multilinear operators: a panorama. Quaest. Math., 34(4):447-478, 2011.

[34] D. Pérez-García. Comparing different classes of absolutely summing multilinear operators. Arch. Math. (Basel), 85(3):258-267, 2005.

[35] A. Pietsch. Operator ideals. North Holland, 1980.

[36] A. Pietsch. Ideals of multilinear functionals (designs of a theory). In Proceedings of the second international conference on operator algebras, ideals, and their applications in theoretical physics (Leipzig, 1983), volume 67 of Teubner-Texte Math., pages 185-199. Teubner, Leipzig, 1984.

[37] S. Reisner. A factorization theorem in Banach lattices and its application to Lorentz spaces. Ann. Inst. Fourier (Grenoble), 31(1):viii, 239-255, 1981.

[38] S. Reisner. On the duals of Lorentz function and sequence spaces. Indiana Univ. Math. J., 31(1):65$72,1982$.

[39] B. Schneider. On absolutely p-summing and related multilinear mappings. Wissenschaftliche Zeitschrift der Brandemburger Landeshochschule, 35(1991):105-117, 1991.

Departamento de Matemática y Ciencias, Universidad de San Andrés, Vito Dumas 284, (B1644BID) Victoria, Buenos Aires, Argentina and COnicet

E-mail address: vero@udesa.edu.ar

Departamento de Matemática - Pab I, Facultad de Cs. Exactas y Naturales, Universidad de Buenos Aires, (C1428EGA) Buenos Aires, Argentina and imas - COniCet.

E-mail address: rvillafa@dm.uba.ar 